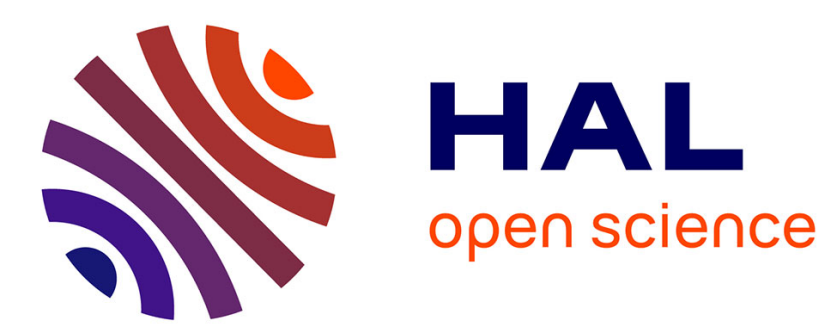

\title{
Etude du couple composé d'insertion du graphite-chlorure de manganèse/ammoniac pour le stockage chimique de la chaleur
}

A. El Atifi, Ph. Touzain, P. Mathonnet

\section{- To cite this version:}

A. El Atifi, Ph. Touzain, P. Mathonnet. Etude du couple composé d'insertion du graphite-chlorure de manganèse/ammoniac pour le stockage chimique de la chaleur. Revue de Physique Appliquée, 1986, 21 (10), pp.603-607. 10.1051/rphysap:019860021010060300 . jpa-00245478

HAL Id: jpa-00245478

https://hal.science/jpa-00245478

Submitted on 1 Jan 1986

HAL is a multi-disciplinary open access archive for the deposit and dissemination of scientific research documents, whether they are published or not. The documents may come from teaching and research institutions in France or abroad, or from public or private research centers.
L'archive ouverte pluridisciplinaire HAL, est destinée au dépôt et à la diffusion de documents scientifiques de niveau recherche, publiés ou non, émanant des établissements d'enseignement et de recherche français ou étrangers, des laboratoires publics ou privés. 


\title{
Etude du couple composé d'insertion du graphite-chlorure de manganèse/ammoniac pour le stockage chimique de la chaleur
}

\author{
A. El Atifi, Ph. Touzain et P. Mathonnet (*) \\ Laboratoire d'Adsorption et Réaction de Gaz sur Solides, UA CNRS 413, ENSEEG. INPG, BP 75 , \\ 38402 St Martin d'Hères, France \\ (*) Service des Transferts Thermiques, C.E.N.G. 85 X, 38041 Grenoble Cedex, France
}

(Reçu le 24 janvier 1986, révisé le 12 juin, accepté le 19 juin 1986)

\begin{abstract}
Résumé. - La réaction entre l'ammoniac gazeux et le chlorure de manganèse inséré dans le graphite en lit fixe a été étudiée expérimentalement en vue de son application dans des systèmes de conversion thermochimique de l'énergie. La cinétique de la réaction ainsi que les échanges thermiques au sein du lit de poudre sont meilleurs que dans le cas du chlorure libre.
\end{abstract}

Abstract. - Experimental study of reaction between ammonia and graphite intercalated manganese chloride has been made in fixed bed, to use it in thermochemical energy conversion systems. Kinetic reaction and heat exchanges in the powder bed are better than for the free chloride.

\section{Introduction.}

Le stockage de la chaleur sous forme d'énergie chimique consiste à utiliser des réactions chimiques réversibles susceptibles de stocker de la chaleur quand elles s'effectuent dans le sens de la réaction endothermique et de la restituer quand elles s'effectuent dans le sens de la réaction exothermique.

Les réactions chimiques entre l'ammoniac gazeux et certains sels métalliques (réactions du type : solide $_{1} \rightleftharpoons$ solide $_{2}+$ gaz) ont souvent été utilisées dans divers systèmes de conversion thermochimique de l'énergie du fait de leurs avantages particuliers :

- forte capacité spécifique de stockage de l'énergie comparée à celles des systèmes utilisant la chaleur sensible ou latente des corps,'

- délais de restitution indifférents et sans pertes thermiques,

- fonctionnement possible dans une large gamme de température, de l'ambiante à $300^{\circ} \mathrm{C}$,

- simplicité de fonctionnement,

- produits utilisés peu coûteux.

En période de stockage, le système reçoit l'énergie thermique d'une source à haute température et l'emmagasine à plus basse température (généralement l'ambiante) en vue d'une utilisation différée. Pendant la décharge du système, l'énergie stockée est restituée à un niveau de température proche de celui de la source.

Des irréversibilités de différentes natures réduisent sensiblement les performances réelles des systèmes utilisant les ammoniacates en lit fixe.
Par exemple, les faibles échanges thermiques et la mauvaise diffusion de l'ammoniac dans les lits de poudre limitent la cinétique des réactions et gênent la production et le transfert de la chaleur.

Afin de trouver une solution à ces problèmes, plusieurs améliorations techniques ont été proposées :

- Pour favoriser les transferts thermiques, le lit de poudre doit être de faible épaisseur ; les échangeurs de chaleur noyés dans le lit devront donc offrir de grandes surfaces d'échange au prix d'une augmentation de la masse thermique du système [1].

- L'utilisation des ammoniacates en suspension dans des liquides inertes a permis une amélioration notable de la cinétique des réactions et des échanges de chaleur $[2,3]$, mais on constate une réduction considérable de la capacité massique et volumique de stockage, ainsi qu'une consommation d'énergie pour l'agitation des suspensions.

- La fluidisation du lit de poudre permettant aux fines particules solides d'être maintenues en suspension dense à l'intérieur d'un courant de fluide ascendant, a nettement amélioré les échanges thermiques et augmenté la vitesse de la réaction [4]. Cependant, mis à part l'emploi d'énergie extérieure pour maintenir l'ammoniac gazeux en circulation, la diminution irréversible de la granulométrie des grains de la poudre et la perte de charges par entraînement qui en résulte, ont rendu la fluidisation de la poudre peu compétitive.

- Les composés d'insertion du graphite (C.I.G.) qui ont été dernièrement proposés [5], pourraient 
fournir une solution aux problèmes liés aux échanges de chaleur et aux cinétiques des réactions dans ces systèmes en permettant de conserver tous leurs avantages.

Dans ce travail, nous avons fait une étude expérimentale sur l'équilibre chimique existant entre l'ammoniac et le composé d'insertion graphite-chlorure de manganèse en vue de son application au stockage chimique de la chaleur.

Après l'indication des méthodes de préparation des C.I.G. et la présentation du dispositif expérimental, nous présenterons les divers résultats expérimentaux obtenus sur les pressions d'équilibre, températures de la poudre, quantités d'ammoniac cyclé, puissances thermiques extraites...

\section{Partie expérimentale.}

2.1 Préparation Des C.I.G. - Pour la préparation des C.I.G. avec les chlorures de métaux de transition tels que $\mathrm{Mn}, \mathrm{Fe}, \mathrm{Co}, \mathrm{Ni}, \mathrm{Cu}, \ldots$ la méthode utilisée consiste à porter à la température $(T)$ un mélange de chlorure et de graphite préalablement déshydraté en présence d'un courant de chlore gazeux à très faible débit sous la pression atmosphérique, pendant un temps $(t)$. La température d'insertion $(T)$ et le temps de la réaction $(t)$ dépendent du produit à insérer. Pour l'insertion du chlorure de manganèse, $T=500{ }^{\circ} \mathrm{C}$ et $t=10 \mathrm{~h}$, pour du graphite naturel de granulométrie comprise entre 10 et $20 \mu \mathrm{m}$. Le produit obtenu est un composé de premier stade de stoechiométrie $\mathrm{C}_{5} \mathrm{MnCl}_{2}$.

2.2 DISPOSITIF EXPÉRIMENTAL. - Le dispositif expérimental utilisé se compose principalement [1] :

- d'une section d'essais calorifugée recevant le

lit de poudre et son échangeur thermique,

- d'une réserve d'ammoniac liquide,

- d'un circuit d'huile thermique caloporteur qui permet de porter les parois de l'échangeur à des températures constantes comprises entre l'ambiante et $300^{\circ} \mathrm{C}$.

L'échangeur du lit d'ammoniacate est un tube à ailettes en acier inoxydable, avec une surface d'échange de $0,55 \mathrm{~m}^{2}$.

Les quantités de chaleur absorbée ou libérée par le lit de poudre sont mesurées en comparant la variation de température de l'huile après traversée de l'échangeur à sa variation de température après passage sur une résistance électrique délivrant une puissance thermique connue.

Un voyant tubulaire associé à la réserve d'ammoniac permet de déterminer la variation de la quantité d'ammoniac absorbé ou désorbé au cours des réactions.

2.3 CONDITIONS OPÉRATOIRES. - La charge de poudre répartie autour de l'échangeur de chaleur correspond à 1,5 mole de chlorure de manganèse insérée dans le graphite $(280 \mathrm{~g}$ de C.I.G.).

Différents essais de déstockage de la chaleur (absorption de l'ammoniac par le C.I.G.) ont été effectués à quatre niveaux de température de l'huile $\left(45^{\circ} \mathrm{C}, 60^{\circ} \mathrm{C}, 80^{\circ} \mathrm{C}, 100^{\circ} \mathrm{C}\right)$, la pression d'ammoniac dans la section étant maintenue constante entre 7,8 et $8,6 \mathrm{~atm}$.

Les phases de stockage (désorption de l'ammoniac) ont été toutes effectuées en portant l'huile à $200{ }^{\circ} \mathrm{C}$ correspondant à une température maximale de $185^{\circ} \mathrm{C}$ du lit de poudre. La pression d'ammoniac a été maintenue à 7,4 atm. 16 cycles de charge et de décharge ont été réalisés sur le montage sans qu'aucune perte de réversibilité n'ait pu être constatée. Dans le but d'étudier la reproductibilité des propriétés thermiques et cinétiques, chaque expérience a été reproduite deux ou trois fois.

$\mathrm{Au}$ cours des phases de charge et de décharge, les paramètres de fonctionnement qui ont été enregistrés sont les suivants :

- la pression d'équilibre d'ammoniac avant la réaction d'absorption,

- la quantité totale d'ammoniac cyclé,

- la variation de la température de la poudre pendant la phase de décharge,

- la variation de la quantité d'ammoniac absorbé ou désorbé pendant les réactions d'absorption et de désorption,

- la variation de la puissance thermique extraite pendant la réaction d'absorption.

\section{Résultats expérimentaux et discussions.}

3.1 QUANTITÉ D'AMMONIAC CYCLÉ. - Lors de la toute première réaction d'absorption, le nombre de moles d'ammoniac fixées par mole de chlorure de manganèse insérée dans le graphite a été d'environ 7,2 moles. Le nombre de moles d'ammoniac cyclées (absorbées ou désorbées) est compris entre 4,6 et 5 moles (Figs. $2 b$ et 3 ).

Ces valeurs sont nettement supérieures aux valeurs trouvées dans la littérature correspondant à l'équilibre de décomposition de l'ammoniacate de chlorure de manganèse seul (6 moles pour la première absorption et 4 moles cyclées) $[1,6]$.

La réaction globale ayant lieu, correspond donc à l'équilibre :

$\mathrm{C}_{5} \mathrm{MnCl}_{2}, 2,4 \mathrm{NH}_{3}+4,8 \mathrm{NH}_{3} \rightleftharpoons \mathrm{C}_{5} \mathrm{MnCl}_{2}, 7,2 \mathrm{NH}_{3}$.

3.2 PRESSION D'ÉQUILIBRE. - La figure 1 représente la variation en fonction de la température de la pression d'ammoniac en équilibre avec le lit du C.I.G. L'équation de la droite correspondante est : $\log _{10} P_{\mathrm{e}}=-2250 / T_{\mathrm{e}}+C$.

Par identification avec l'équation

$$
\ln P=-\Delta H / R T+\Delta S / R T,
$$

on déduit une valeur de $43,3 \mathrm{~kJ} /$ mole de $\mathrm{NH}_{3}$ pour l'enthalpie de fixation d'une molécule d'ammoniac sur le C.I.G. Cette valeur est voisine de celle trouvée avec le même dispositif expérimental pour $\mathrm{MnCl}_{2}$ pur $(42,6 \mathrm{~kJ} / \mathrm{mole})$. 


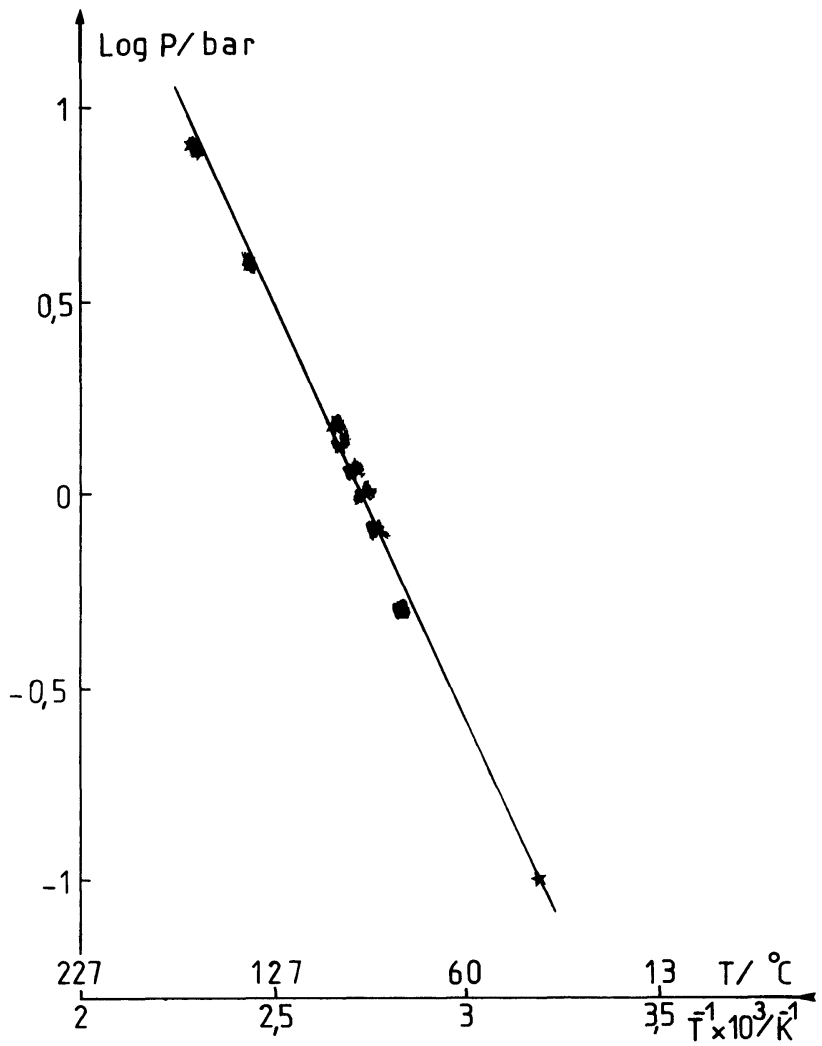

Fig. 1. - Diagramme $\log P, 1 / T$ pour le couple C.I.G.$\mathrm{NH}_{3}$.

[Diagram $\log P, 1 / T$ for the $\mathrm{NH}_{3}$-G.I.C. couple.]

3.3 TEMPÉRATURE DE LA POUDRE PENDANT L'ABSORPTION. - La figure 2 a représente la variation de la température de la poudre du C.I.G. au cours de la réaction d'absorption effectuée à partir de différents niveaux de température. La température du fluide thermique a été maintenue constante pendant toute l'expérience.

En comparant ces courbes entre elles, on remarque d'une part que la durée de la réaction d'absorption diminue avec la température initiale de la poudre, d'autre part, la valeur maximale de la température atteinte par la poudre est d'autant plus grande que sa température initiale est élevée. Le tableau I rassemble ces résultats.

3.4 CINÉTIQUE DES RÉACTIONS D'ABSORPTION ET DE DÉSORPTION. - La variation en fonction du temps de la quantité $n$ d'ammoniac absorbé par le lit de poudre est donnée sur la figure $2 b$.

En comparant le temps total de réaction $t$ correspondant aux différentes conditions expérimentales, on vérifie bien que les réactions d'absorption sont plus rapides lorsque la température initiale du lit de poudre est plus basse. Ces valeurs sont aussi données dans le tableau I.

La variation en fonction du temps de la quantité $n$ d'ammoniac désorbé pendant les phases de charge est représentée sur la figure 3. Les différents essais effectués dans les mêmes conditions montrent une bonne reproductibilité de l'expérience. Le temps total de la charge est de $60 \mathrm{~min}$.
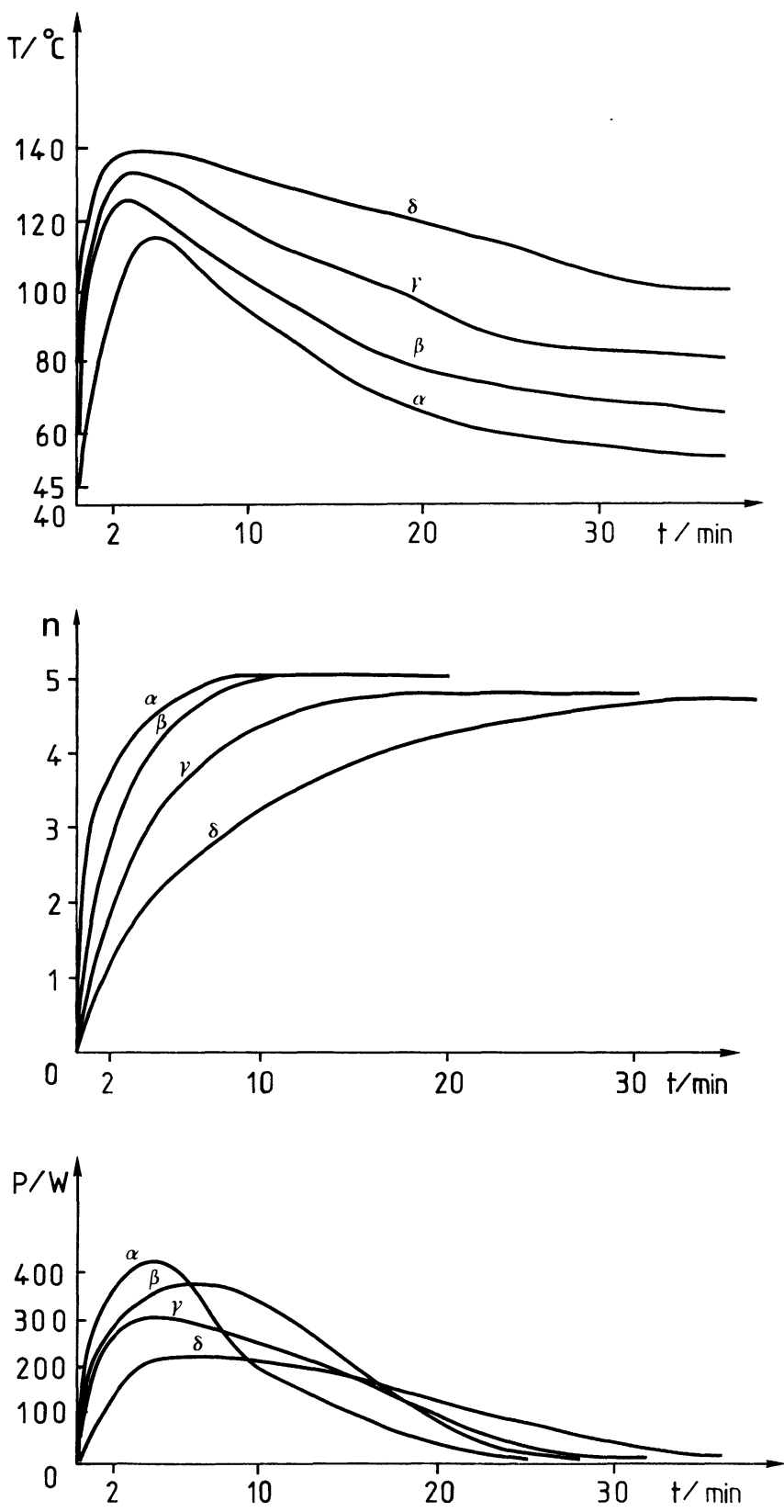

Fig. 2. - Variation en fonction du temps : a) de la température $T$ du lit de poudre, b) du nombre de moles $n$ d'ammoniac absorbées rapporté à une mole de C.I.G., c) de la puissance thermique $P$ extraite, pour différentes températures initiales du lit de poudre : $T_{\mathrm{i}}(\alpha)=45^{\circ} \mathrm{C}$, $T_{\mathrm{i}}(\beta)=60^{\circ} \mathrm{C}, T_{\mathrm{i}}(\gamma)=80^{\circ} \mathrm{C}, T_{\mathrm{i}}(\delta)=100{ }^{\circ} \mathrm{C}$.

[Relationship between time and : a) powder bed temperature $T$, b) number of absorbed moles $n$ of ammonia related to one mole of G.I.C., c) extracted heat power $P$, for different initial temperatures of the powder bed: $T_{\mathrm{i}}(\alpha)=45^{\circ} \mathrm{C}, \quad T_{\mathrm{i}}(\beta)=60^{\circ} \mathrm{C}, \quad T_{\mathrm{i}}(\gamma)=80^{\circ} \mathrm{C}$, $T_{\mathrm{i}}(\delta)=100{ }^{\circ} \mathrm{C}$.] 
Tableau I. - Résumé des résultats expérimentaux.

[Abstract of experimental results].

\begin{tabular}{|c|c|c|c|c|c|c|}
\hline$T_{i} /{ }^{\circ} \mathrm{C}$ & $t / \min$ & $T_{\max } /{ }^{\circ} \mathrm{C}$ & $\Delta T_{\max } /{ }^{\circ} \mathrm{C}$ & $P_{\max } / \mathrm{W}$ & $h / \mathrm{W} \mathrm{m}^{-2}{ }^{\circ} \mathrm{C}$ & $Q / \mathrm{kJ}$ \\
\hline 45 & 8 & 115 & 70 & 450 & 11,6 & 260 \\
60 & 10 & 125 & 65 & 400 & 11,1 & 260 \\
80 & 16 & 134 & 54 & 300 & 10,1 & 270 \\
100 & 30 & 140 & 40 & 220 & 10 & 220 \\
\hline
\end{tabular}

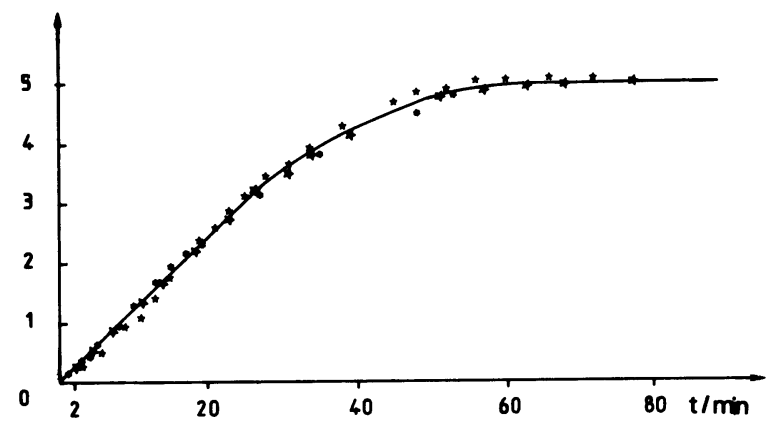

Fig. 3. - Variation en fonction du temps du nombre de moles $n$ d'atmmoniac désorbé rapporté à une mole de C.I.G. pour quelques cycles.

[Number of desorbed mole $n$ of ammonia related to one mole of C.I.G. as a function of time for some cycles.]

Ces valeurs des temps de réaction sont 2 à 3 fois plus faibles que celles trouvées avec $\mathrm{MnCl}_{2}$ pur (Tableau II) ou avec les chlorures métalliques purs en général [3]. Cette nette amélioration de la vitesse de réaction provient du fait de la répartition à l'échelle atomique du chlorure de manganèse entre les plans graphitiques qui facilite la diffusion externe et interne de l'ammoniac.

3.5 PUISSANCE THERMIQUe EXTRAITE. - La variation en fonction du temps de la puissance thermique $P$ extraite du lit de poudre est donnée pour chaque niveau de température sur la figure 2c. Dans le tableau I, nous avons rapporté les valeurs des maxima de ces courbes, des coefficients d'échange thermique et des quantités de chaleur restituée.
Grâce à un bón calorifugeage au niveau de la section d'essais, on a pu obtenir des résultats significatifs. Ils nous permettent de calculer un coefficient d'échange thermique $h$ de $10,7 \mathrm{~W} / \mathrm{m}^{2}{ }^{\circ} \mathrm{C}$ (avec $h=P_{\max } / S . \Delta T_{\max }, S$ la surface d'échange) au lieu de 6 avec $\mathrm{MnCl}_{2}$ pur (Tableau II).

La valeur moyenne de la chaleur restituée $Q$ à l'aide du fluide caloporteur circulant à l'intérieur de l'échangeur est de $257 \mathrm{~kJ}$ pour les 1,5 mole de C.I.G. présente dans le réacteur (l'équivalent de $755 \mathrm{~kJ} / \mathrm{kg}$ ) soit $80 \%$ de la chaleur théorique $(913 \mathrm{~kJ} / \mathrm{kg})$.

Si on tient compte des pertes thermiques inévitables dues à l'échauffement du gaz ammoniac et de la masse thermique du réacteur, ces résultats sont assez satisfaisants, ils montrent bien une amélioration des échanges thermiques au sein du lit de poudre. Ceci est une conséquence directe de la bonne conductivité thermique des C.I.G.

\section{Conclusion.}

La substitution du chlorure de manganèse par son composé d'insertion du graphite dans le système de stockage chimique de la chaleur présente donc les avantages spécifiques suivants :

- amélioration de la cinétique de la réaction grâce à une meilleure diffusion de l'ammoniac,

- augmentation de la réactivité du chlorure (absorption de 7 moles d'ammoniac au lieu de 6 avec le chlorure pur) ce qui conduit à une capacité de stockage plus importante,

Tableau II. - Quelques résultats à titre comparatif concernant le couple $\mathrm{MnCl}_{2}-\mathrm{NH}_{3}$ sans graphite dans le même dispositif expérimental et dans les mêmes conditions que celles du tableau $\mathrm{I}$, rapportés à 1,5 mole de $\mathrm{MnCl}_{2}$.

[Some comparative experimental results about $\mathrm{NH}_{3}-\mathrm{MnCl}_{2}$ couple without graphite in the same apparatus and for the same conditions than table I, refer to 1.5 mole of $\mathrm{MnCl}_{2}$ ].

\begin{tabular}{|c|c|c|c|c|c|c|}
\hline$T_{i} /{ }^{\circ} \mathrm{C}$ & $t / \min$ & $T_{\max }{ }^{\circ} \mathrm{C}$ & $\Delta T_{\max }{ }^{\circ} \mathrm{C}$ & $P_{\max } / \mathrm{W}$ & $h / \mathrm{W} \mathrm{m}^{-2}{ }^{\circ} \mathrm{C}$ & $Q / \mathrm{kJ}$ \\
\hline 60 & 30 & 120 & 60 & 254 & 6 & 180 \\
100 & 50 & 132 & 32 & 136 & 6 & 120 \\
\hline
\end{tabular}


- amélioration des échanges thermiques au sein du lit de poudre permettant ainsi un bon écoulement de la chaleur vers l'échangeur thermique.

Toutes ces améliorations pourront être apportées a priori à tous systèmes thermochimiques fonctionnant sur ce principe avec n'importe quel chlorure de métal de transition donnant un composé d'insertion du graphite. Seules changeraient les conditions de fonctionnement.

\section{Remerciements.}

Les auteurs remercient la Société «Le Carbone Lorraine " pour l'approvisionnement en graphite et Messieurs P. Blum, J. Michel et C. Moreau pour la réalisation d'expériences au C.E.N.G.

\section{Bibliographie}

[1] Elberg, S., Mathonnet, P., Revue Phys. Appl. 17 (1982) 585.

[2] Wentworth, W. E., Batten, C. F., Ayala, J. A. et Alcala, R., J. Phys. Chem. 86 (1982) 3483.

[3] Mauran, S., Crozat, G., Bodiot, D. et SpinNer, B., J. Chim. Phys. 82 (1985) 791.
[4] Romero, J., Thèse de $3^{\mathrm{c}}$ cycle, INP Grenoble (1982).

[5] Touzain, P., Michel, J., Blum, P., Synthetic Metals 8 (1983) 313.

[6] Remy, F., Revue Chim. Miné. 2 (1986) 693. 